\title{
Identification of Socio-Demographic, Behavioral Patterns and Their Relationship with HIV Status among Female Sex Workers
}

\author{
Venkaiah Kodavalla1 ${ }^{*}$, Hemalatha Rajkumar', Harikumar Rachakulla1, \\ Paleswara Vara Prasad Saride1, Srinivasan Kallam², \\ Brahmam Narsimhachari Veera Ginnela1 \\ ${ }^{1}$ National Institute of Nutrition, Hyderabad, India \\ ${ }^{2}$ FHI30, New Delhi, India \\ Email: ${ }^{*}$ kodalivenkaiah@yahoo.com
}

Received 7 February 2015; accepted 25 March 2015; published 26 March 2015

Copyright (C) 2015 by authors and Scientific Research Publishing Inc.

This work is licensed under the Creative Commons Attribution International License (CC BY). http://creativecommons.org/licenses/by/4.0/

(c) (i) Open Access

\section{Abstract}

The prevalence of HIV in high risk population is influenced significantly the behavioral and sociodemographic characteristics. However, considering the complexity of behavior among female sex workers, the relationship between a particular behavioral pattern and the HIV status of this "at risk" population assumes significance. Data generated in a community-based cross-sectional study earlier carried out to assess the prevalence estimates, at district level, of HIV status in eight districts of State of Andhra Pradesh, India was used to carry out factor analysis to explore the role of demographic and behavioral pattern and their relationship with the HIV status among female sex workers. Data on 3083 female sex workers in the study revealed that there existed nine patterns among demographic and behavioral characteristics, which explained $62 \%$ of the total variation through factor analysis. Further, cluster analysis was performed to identify the groups of individuals having similar characteristics. Two of those clusters had sizeable numbers having similar characteristics. FSWs belonging to cluster 2 had significantly high risk factors compared with Cluster 1. The overall prevalence of HIV was $11.4 \%$ (10.6\% in cluster 1 and $15.9 \%$ in cluster 2$)$ among high risk population. There exists a strong relationship between behavioral patterns and HIV positive.

\section{Keywords}

Behavioral Patterns, Community-Based Cross-Sectional Studies, Principal Component Analysis (PCA), Factor Analysis, Cluster Analysis, HIV Status

\footnotetext{
*Corresponding author.
}

How to cite this paper: Kodavalla, V., Rajkumar, H., Rachakulla, H., Saride, P.V.P., Kallam, S. and Ginnela, B.N.V. (2015) Identification of Socio-Demographic, Behavioral Patterns and Their Relationship with HIV Status among Female Sex Workers. World Journal of AIDS, 5, 41-49. http://dx.doi.org/10.4236/wja.2015.51005 


\section{Introduction}

India, the second-most populous country in the world, has been experiencing a highly varied HIV epidemic, in the past two decades. HIV in India is concentrated mainly among "high-risk" populations such as Female Sex Workers (FSWs), Men having Sex with Men (MSM) and Injecting Drug Users (IDUs). Targeted interventions (TIs) among these populations have been a major aspect of India's National AIDS Control Programme (NACP). The recent HIV estimates showed a reduction in the adult HIV prevalence from $0.41 \%$ in the year 2000 , through $0.36 \%$ in 2006 to $0.31 \%$ in 2009. The estimated number of new annual HIV infections declined from 2.7 lakh in 2000 to 1.2 lakh in 2009 [1].

New infections reduced by $50 \%$ during this period. Therefore sustained focus on prevention is envisaged by NACO.

Andhra Pradesh (AP) has been categorised as "A" with HIV prevalence among FSW $>1 \%$ and the state remains to be critical in the spread \& control of the epidemic. The state has the second highest estimated adult HIV prevalence of $0.90 \%$ after Manipur (at 1.40\%). While India has an estimated 24 lakh people living with HIV/AIDS in 2009, AP tops the list of states with about 5 lakh people living with HIV/AIDS; followed by 4.2 lakh in Maharashtra, 2.5 lakh in Karnataka and 1.5 lakh in Tamil Nadu. Of the estimated people living with HIV/AIDS, 9.3 lakh (39\%) are women [1].

HIV prevalence among Female Sex Workers (FSW) in Andhra Pradesh increased from 7.3\% in 2006 to 9.7\% in 2007. Sentinel surveillance data of 2007 also depicted Andhra Pradesh as the only state with $>1 \%$ prevalence among ANC attendees [2]. (HIV Sentinel Surveillance and HIV Estimation in India 2007. A Technical BriefNACO.)

Targeted interventions by the Andhra Pradesh AIDS Control Society (APSACS) and Avahan by Bill and Melinda Gates Foundation (BMGF) are the two major HIV/AIDS prevention programs currently in operation in the state [3]. The state government (in partnership with NACO) has been implementing prevention, care and treatment programs since 1999, which have resulted in significant gains. However, the epidemic continues to be of serious concern, as recent surveillance rounds reveal that, across Andhra Pradesh, prevalence levels continue to display an upward trend, pointing to the need for intensifying preventive intervention programmes.

In this backdrop, the present paper utilizes the "Integrated Behavioral and Biological Assessment" (IBBA) data to analyze and identify the demographic, behavioral and biological patterns among FSWs that contribute or relate to the HIV prevalence in the State of Andhra Pradesh [4].

\section{Objective}

The objective of the present communication is to identify behavioral patterns of FSWs and study their relationship with HIV status.

\section{Methodology}

\subsection{Sampling \& Sample Size}

Considering consistent use of condom by 50\% FSWs with the clients during round 1 with $15 \%$ increase during round 2, with $95 \%$ CI, 90\% power and 1.7 as design effect the required sample size is 385 , rounded-off to 400 , which adds up to 3200 FSWs for 8 districts [4]. Sampling frame consisting of different sites of solicitation was developed for each district, a fortnight before commencing the actual survey. Probability sampling method with varied sampling approaches was used to select the site as Primary Sampling Units (PSUs) at the first stage. Conventional Cluster Sampling (CCS) as well as Time-Location Cluster Sampling (TLCS) approaches were adopted, based on the characteristics of the different FSW sub-groups in various districts [5]. FSWs who operated from home were grouped as "home based", while those who operated from other places/fixed locations such as brothel, dabha, lodge, or rented room were grouped as "brothel based". The sex workers who were mobile and operated in public places such as street, bus stand, railway station and others were grouped as public place-based sex workers. The CCS approach was used for selecting brothel and home-based FSWs, while TLCS method was used to select public place-based sex workers [5].

\subsection{Coverage}

The districts of Hyderabad, Karimnagar and Warangal from Telangana Region, Visakhapatnam, East Godavari, 
Guntur and Prakasam from Costal Region, Chittoor District from Rayalseema Region were covered in the survey, which were under the Avahan interventions, having a high number of Key populations (KPs), diversity among different socio-cultural strata in the region and with a goal of modeling Avahan's impact in different types of settings. A total of 3083 FSWs were covered from 8 districts in the State.

For the study, FSWs were operationally defined as "any female, $\geq 18$ years, either brothel based (working/ living/operating in red light/brothel areas), home based or public place based (soliciting male clients on the street or in other non-brothel settings), who sold sex in exchange for cash at least once in the last one month".

The potential risk variables considered in the present analysis included demographic variables such as 1) age 2) literacy, 3) marital status 4) age of initiation of sex work and duration in sex work, the behavioral variables such as 1) having regular clients, 2) having regular unpaid partners, 3) having other regular unpaid partners, 4) having occasional clients, 5) wanted to use condom but could not use due to several reasons, 6) dependency on sex work, 7) had sex during travel, 8) client volume per week, 9) carrying condom at the time of interview, 10) exposed to any interventions, 11) correct knowledge of HIV/AIDS, 12) obtained condom from peer educator and the biological parameters such as 1) history of having any STI during past 12 months and suffering from any STI (syphilis or NG or CT) at the time of interview.

The study was approved by the Health Ministry Screening Committee, Government of India, Family Health International's Protection of Human Subjects Committee and Andhra Pradesh State AIDS Control Society, the Government of Andhra Pradesh. In addition, the Scientific Advisory Committee of National AIDS Research Institute (NARI) approved the IBBA protocol, and National Institute of Nutrition (NIN) Ethics Committees reviewed and approved the consent forms, study instruments, Standard Operating Procedures (SOPs) and field manuals.

\subsection{Statistical Analysis}

The percentage distributions of different demographic, behavioral and biological variables were calculated. Behavioral patterns were obtained by exploratory factor analysis for 20 potential risk variables considering simultaneously [6]. The principal component analysis (PCA) technique, followed by factor analysis was used to transform a large set of correlated variables into smaller sets of non-correlated variables, called principal components or factors [7]. The factor analysis of the data objectively points out characteristics those are clustered. The aim of this technique is to identify the underlying structure in data matrix, by summarizing and consigning data to arrive at a systematic measurement of the behavior. To summarize data, factor analysis requires dimension that, when interpreted and understood, describes it in terms of a much smaller number of significant variables than a host of variables.

For the applicability of factor analysis, the uniformity of sample was tested by examining the distribution of variables in a loading plot, contrasting the value observed against those expected in a normal distribution which was verified by Kaiser-Meyer-Olkin (KMO) measurement of adequacy [8]. A KMO value of more than 0.50 was considered acceptable. The presence of correlations between demographic, behavioral and biological variables was tested using the Bartlett test of sphericity (homogeneity of variance). The Bartlett test statistic is approximately distributed with chi-square and was accepted at a minimum significance level of $p<0.05$. Principle Component Analysis was used for extraction of factors and orthogonal rotation (varimax option) to derive non-correlated factors, to minimize the number of indicators that have high loading on one factor. The first factor extracted is the one that accounts for the maximum possible variance in the dataset. The second component, independent of the first, will be the one that explains the largest possible share of the remaining variance and so on, without the components being correlated with each other. While deriving behavioral patterns for FSWs dataset, the variable, viz., current HIV status is not included in the analysis as it is intended to study the relationship between behavioral patterns vis-à-vis HIV status.

Kaiser criterion, namely Eigen value of $>1.0$, is widely-used for choosing the number of factors in the factor analysis. It was also based on the Eigen plot (scree plot), which shows the total variance associated with each other.

Cluster analysis was performed to identify relatively homogeneous groups of cases based on the factor scores derived through factor analysis, using an algorithm that can handle large number of cases [9]. However, number of clusters would depend on the number of cases fall in different clusters. The prevalence of HIV was considered to name and label the cluster. Correctness of labeling of cluster was verified by testing the significant difference between the clusters on the back ground characteristics of the cases. 
Discriminant function analysis was used for studying the relationship between the demographic, socioeconomic and behavioral variables with HIV status. The factor scores obtained by the factor analysis for these variables were considered as continuous independent variables, and HIV status of the female sex workers was considered as dichotomous dependent variable. Sensitivity and Specificity of the discriminant function was also calculated. The statistical analysis was performed using SPSS [10].

\section{Results}

The distribution of FSWs according to demographic and behavioral characteristics is presented in Table 1. Majority (42\%) of FSWs solicit their clients in public places. About 19\% FSWs were in the age group of $<25$ years,

Table 1. Frequency (\%) distribution of FSWs according to their demographic, Behavioral and biological profile ( $\mathrm{n}=3083)$.

\begin{tabular}{|c|c|c|}
\hline Variable & Category & $\%$ \\
\hline \multirow[t]{4}{*}{ Age (years) } & $<25$ & 18.7 \\
\hline & $25-29$ & 26.1 \\
\hline & $30-34$ & 20.7 \\
\hline & $35+$ & 34.6 \\
\hline \multirow[t]{4}{*}{ Age at start of sex work (years) } & $<20$ & 18.2 \\
\hline & $20-24$ & 32.0 \\
\hline & $25-29$ & 26.4 \\
\hline & $30+$ & 23.4 \\
\hline \multirow[t]{4}{*}{ Duration of sex work (years) } & $0-1$ & 14.6 \\
\hline & $2-4$ & 35.9 \\
\hline & $5-9$ & 30.4 \\
\hline & $10+$ & 19.1 \\
\hline \multirow[t]{3}{*}{ Client volume per week } & $<5$ & 10.4 \\
\hline & $5-9$ & 38.1 \\
\hline & $10+$ & 51.5 \\
\hline \multirow[t]{3}{*}{ Usual place of solicitation } & Home & 38.3 \\
\hline & Brothel/lodge/daba & 20.3 \\
\hline & Public places & 41.5 \\
\hline Literacy status & Literate & 42.3 \\
\hline Depend on sex work & Yes & 49.3 \\
\hline Have other regular un paid partners & Yes & 72.8 \\
\hline Had sex during travel & Yes & 7.7 \\
\hline Having regular clients & Yes & 83.3 \\
\hline Having unpaid regular partners & Yes & 72.8 \\
\hline Consistent condom use with occasional clients & Yes & 80.9 \\
\hline Wanted to use condom but could not use & Yes & 17.2 \\
\hline Carrying condom at the time of interview & Yes & 46.6 \\
\hline Obtained condom from peer educator & Yes & 60.8 \\
\hline Exposed to intervention & Yes & 75.8 \\
\hline Ever taken HIV test & Yes & 73.5 \\
\hline History of having STI during past 12 months & Yes & 32.7 \\
\hline HIV positive & Yes & 11.4 \\
\hline Presently suffering from any STI (NG, CT, syphilis) & Yes & 10.1 \\
\hline Having correct knowledge about HIV & Yes & 0.9 \\
\hline
\end{tabular}


about $47 \%$ were in the age group of 25 - 34, while 35\% were in the age group of 35+ years. Majority (50\%) of FSWs started commercial sex work before 25 years about $50 \%$ reported a duration of $>5$ years of sex work, While half of the FSWs reported that their volume of clients was $>10$ per week.

About $42 \%$ of FSWs were literates and 56\% were married and staying with their family. Majority of FSWs had regular unpaid partners (72.8\%) and regular clients (83.3\%). Majority of the FSWs (81\%) reported that they were using condom consistently with occasional clients, about $17.2 \%$ reported that they wanted to use condom but could not use due to various reasons and very few (7.7\%) reported had sex during last travel. About $47 \%$ of FSWs were carrying condom at the time of survey, while $61 \%$ reported that they obtained the condom from peer educator. About 33\% of FSWs reported the history of having any STI (NG, CT, and Syphilis), while 10.1\% were currently suffering from STIs. Only $1 \%$ of the FSWs had correct knowledge on HIV. While majority of FSWs (73.5\%) reportedly undergone HIV testing, 11.4\% of them were HIV positive.

Analysis reveals that nine components for high risk population were extracted by factor analysis using Principal Component Analysis with varimax rotation for demographic, socioeconomic and behavioral variables (Table 2). These nine components (factors) in the initial solution have an Eigen value over 1, and they account for about $62 \%$ of the observed variation in the behavioral pattern among female sex workers. The parallel line to horizontal at Eigen value equaling to 1 in scree plot showed that nine factors will be extracted for this population (Figure 1). The first factor, which accounted for $10.9 \%$ of the total variance among the female sex workers was labeled as having unpaid regular partners. High factor loading observed for those FSWs who are either married or having a regular unpaid male partner characterized these factors. The second factor explained $9.2 \%$ of the total variance and was labeled as exposure to intervention (exposed to any type of intervention and obtained condom from peer educator employed by the NGOs working in that area). The third factor which accounted for $7.8 \%$ of the total variance was characterized by the age of the FSW and duration in sex work was labeled as age \& duration of sex work. The fourth factor explained $6.8 \%$ of the total variance was done labeled as correct knowledge of HIV/AIDS and getting HIV testing done voluntarily. The fifth factor accounted for $6.0 \%$ of the total variance and characterized having any STIs for the past 12 months and wanted to use condom but could not use and it was labeled as suffering from STIs. The sixth factor explained $5.4 \%$ of the total variance and was labeled as dependency (majorly depend up on sex work and the place of solicitation). The seventh factor accounted for $5.2 \%$ of the total variance and was characterized by single variable namely having regular clients who pay every

Table 2. Rotated component matrix for socio-demographic, behavioral and biological parameters.

\begin{tabular}{|c|c|c|c|c|c|c|c|c|c|}
\hline \multicolumn{10}{|c|}{ Rotated component matrix ${ }^{a}$} \\
\hline & \multicolumn{9}{|c|}{ Component } \\
\hline & 1 & 2 & 3 & 4 & 5 & 6 & 7 & 8 & 9 \\
\hline Had sex during travel & -0.087 & -0.263 & 0.202 & 0.329 & 0.199 & 0.019 & 0.425 & 0.021 & 0.110 \\
\hline Obtained condom from PE & 0.005 & 0.774 & 0.048 & 0.074 & -0.135 & 0.005 & 0.093 & -0.001 & 0.021 \\
\hline Wanted to use condom but could not & -0.001 & -0.211 & 0.169 & -0.078 & 0.573 & 0.273 & -0.079 & -0.042 & -0.019 \\
\hline Have occasional clients & 0.034 & 0.153 & 0.011 & 0.266 & 0.020 & 0.038 & -0.462 & 0.546 & 0.021 \\
\hline Have regular clients & 0.047 & 0.186 & 0.036 & -0.005 & -0.060 & -0.080 & 0.680 & 0.086 & -0.108 \\
\hline Have regular unpaid partners & 0.971 & 0.035 & -0.013 & 0.020 & 0.031 & 0.017 & 0.026 & -0.001 & -0.018 \\
\hline Have other regular unpaid partners & 0.109 & -0.212 & -0.188 & 0.187 & 0.399 & -0.007 & 0.084 & 0.272 & -0.153 \\
\hline Ever taken HIV test & 0.019 & 0.243 & 0.066 & 0.642 & 0.077 & -0.006 & -0.011 & -0.068 & -0.004 \\
\hline Literate & 0.021 & -0.196 & -0.295 & 0.311 & -0.056 & -0.492 & 0.173 & -0.035 & -0.155 \\
\hline Dependent on sex work & 0.088 & 0.140 & -0.043 & 0.001 & 0.255 & 0.611 & 0.169 & -0.016 & -0.238 \\
\hline Solicitation place & -0.021 & -0.227 & -0.065 & 0.261 & -0.298 & 0.642 & -0.070 & 0.028 & 0.135 \\
\hline Carrying condom & 0.010 & 0.308 & -0.059 & 0.013 & -0.044 & 0.216 & 0.423 & 0.079 & 0.175 \\
\hline Had any STI in past 12 months & 0.003 & 0.107 & -0.126 & 0.066 & 0.723 & -0.121 & -0.022 & -0.036 & 0.121 \\
\hline Any STI (syph or NG or CT) & -0.021 & -0.006 & -0.019 & -0.007 & 0.056 & -0.009 & 0.002 & 0.002 & 0.916 \\
\hline Exposed to any intervention & 0.078 & 0.719 & 0.096 & 0.272 & 0.084 & 0.019 & 0.097 & 0.095 & -0.057 \\
\hline Marital status & 0.972 & 0.039 & 0.000 & 0.037 & 0.013 & 0.024 & -0.007 & 0.001 & -0.015 \\
\hline Correct knowledge of HIV/AIDS & 0.035 & 0.073 & -0.073 & 0.720 & -0.025 & 0.040 & 0.001 & 0.074 & -0.007 \\
\hline Age & -0.012 & 0.101 & 0.808 & -0.033 & 0.001 & 0.184 & -0.079 & -0.056 & -0.097 \\
\hline Clients per week & -0.027 & 0.034 & 0.030 & -0.090 & -0.025 & 0.004 & 0.191 & 0.831 & 0.010 \\
\hline Duration in sex work & 0.006 & 0.008 & 0.776 & 0.037 & -0.077 & -0.166 & 0.157 & 0.073 & 0.063 \\
\hline
\end{tabular}

Extraction method: principal component analysis. Rotation method: varimax with kaiser normalization. ${ }^{\mathrm{a}}$ Rotation converged in 16 iterations. 


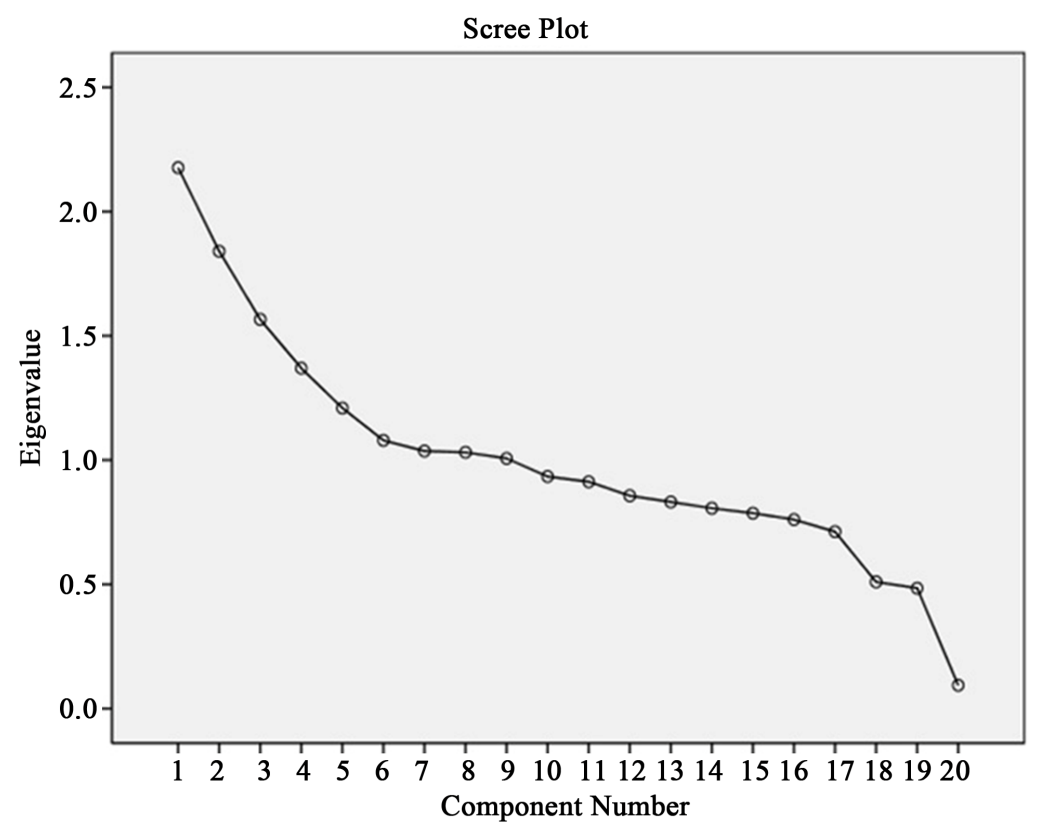

Scree plot showing Eigen values for each of the Socio-demographic, Behavioral and Biological components, extracted by factor analysis.

Figure 1. Socio-demographic, behavioral and biological characteristics of FSWs.

time which was labeled as regular clients. The eighth factor also explained $5.2 \%$ of the total variance and was labeled as having occasional clients (having occasional clients and the volume of these occasional clients per week). The ninth and the last factor explained 5.0\% of the total variance and was labeled as positive for STIs (at the time of survey positive).

In the present analysis, two clusters were formed with sizeable numbers in each of the cluster for further analysis. Cluster 1 was formed with 2668 subjects and cluster 2 with 415 subjects. The distributions of the FSWs according to their back ground characteristics in these two clusters were tabulated and look for significant difference in the proportions are presented in Table 3 . The data revealed significant $(\mathrm{p}<0.01)$ differences in all the variables studied except dependency on sex work, having other regular unpaid partners, ever taken HIV test and having correct knowledge regarding HIV/AIDS between clusters (formed on the basis of behavioral patterns). The FSWs belongs to cluster 2 had higher age, about 35\% had 10+ years of sex work, 24\% had started at early age (<20 years). The literacy status of FSWs of cluster 2 was higher (48\% vs 42\%) had sex during travel (1\% vs $57 \%$ ), having regular clients (82\% vs $94 \%$ ) and consistent condom use with occasional clients (83\% vs $70 \%)$ compared to cluster 1 . Twenty eight percent of FSWs of cluster 2 reported that they want to use condom but could not use due to various reasons during last sex act, 54\% were carrying condom at the time of interview and $51 \%$ of respondents were obtained condom from peer educator compared to $62 \%$ which is lower than that of FSWs belongs to cluster 1. Exposed to intervention by the FSWs belong to cluster 2 was less (68\% against 77\%) of cluster 1. Significantly high proportion of FSWs of cluster 2 against of cluster 1 had the history of STIs (43\% vs $31 \%$ ) as well as currently suffering $28 \%$ vs $7 \%$ ). The overall prevalence of HIV was $11.4 \%$ and the prevalence was significantly high (15.9\%) among FSWs belongs to cluster 2 compared to cluster 1 (10.6\%). These findings indicate that the FSWs of cluster-2 had different socio-demographic, behavioral characteristics which are likely be the risk factors for STIs and HIV positivity and can be named as relatively bad cluster.

The factor scores obtained for these populations through factor analysis were used for the prediction and classification of subjects having HIV based on behavioral patterns. The present data showed a considerable relationship between the socio demographic, behavioral and biological patterns and the HIV status. About $68 \%$ of FSWs were correctly classified as they were positive or negative for HIV test with sensitivity of 51.4\% and specificity of 70\% (Table 4). The behavioral patterns that are extracted from the data obtained in the study may not be applicable to other populations with altogether different behavioral characteristics. 
Table 3. Distribution (\%) of FSWs by their background characteristics by clusters $1 \& 2$.

\begin{tabular}{|c|c|c|c|}
\hline Variable & Category & Cluster-1 (n = 2668) & Cluster-2 $(n=415)$ \\
\hline \multirow[t]{4}{*}{ Age (years) ${ }^{* *}$} & $<25$ & 19.1 & 15.9 \\
\hline & $25-29$ & 26.7 & 22.2 \\
\hline & $30-34$ & 21.0 & 18.3 \\
\hline & $35+$ & 33.2 & 43.6 \\
\hline \multirow[t]{4}{*}{ Duration of sex work (years) $^{* *}$} & $0-1$ & 15.9 & 5.8 \\
\hline & $2-4$ & 37.1 & 28.0 \\
\hline & $5-9$ & 30.2 & 31.8 \\
\hline & $10^{+}$ & 16.8 & 34.5 \\
\hline \multirow[t]{4}{*}{ Age at start of sex work (years) ${ }^{* *}$} & $<20$ & 17.2 & 24.3 \\
\hline & $20-24$ & 31.7 & 34.0 \\
\hline & $25-29$ & 26.8 & 23.5 \\
\hline & $30+$ & 24.2 & 18.3 \\
\hline \multirow[t]{3}{*}{ Client volume per week $^{* *}$} & $<5$ & 9.2 & 18.4 \\
\hline & $5-9$ & 38.9 & 32.9 \\
\hline & $10^{+}$ & 51.9 & 48.8 \\
\hline \multirow[t]{3}{*}{ Typology $^{* *}$} & Home based & 19.7 & 19.5 \\
\hline & Brothel/lodge dhabha & 9.3 & 11.6 \\
\hline & Public place & 71.0 & 68.9 \\
\hline Literacy status & Yes & 41.5 & $48.0^{* *}$ \\
\hline Depend up on sex work & Yes & 49.6 & 48.0 \\
\hline Having other regular un paid partners & Yes & 23.0 & 22.7 \\
\hline Had sex during travel & Yes & 1 & $57^{* *}$ \\
\hline Having regular clients & Yes & 82 & $94^{* *}$ \\
\hline Having unpaid regular partners & Yes & 74 & $63^{* *}$ \\
\hline Consistent condom use with occasional clients & Yes & 82.6 & $69.9^{* *}$ \\
\hline Wanted to use condom but could not use during last sex & Yes & 16 & $28^{* *}$ \\
\hline Carrying condom at the time of interview & Yes & 45.5 & $53.5^{* *}$ \\
\hline Obtained condom from peer educator & Yes & 62.3 & $51.3^{* *}$ \\
\hline Exposed to intervention & Yes & 77 & $68^{* *}$ \\
\hline Ever taken HIV test & Yes & 73.3 & 74.7 \\
\hline History of having STI in past 12 months & Yes & 31.1 & $43.1^{* *}$ \\
\hline HIV positivity & Yes & 10.6 & $15.9^{* *}$ \\
\hline $\begin{array}{l}\text { Presently suffering from any STI } \\
\text { (NG, CT, Syphilis) }\end{array}$ & Yes & 7.4 & $27.5^{* *}$ \\
\hline Having correct knowledge about HIV & Yes & 1.0 & 2.0 \\
\hline
\end{tabular}

$\chi^{2} \cdot{ }^{* *} \operatorname{Sig} \mathrm{p}<0.01$ 
Table 4. Classification of subjects with HIV and without HIV using observed HIV and predicted HIV using scores derived from factor analysis for demographic \& behavioral characteristics.

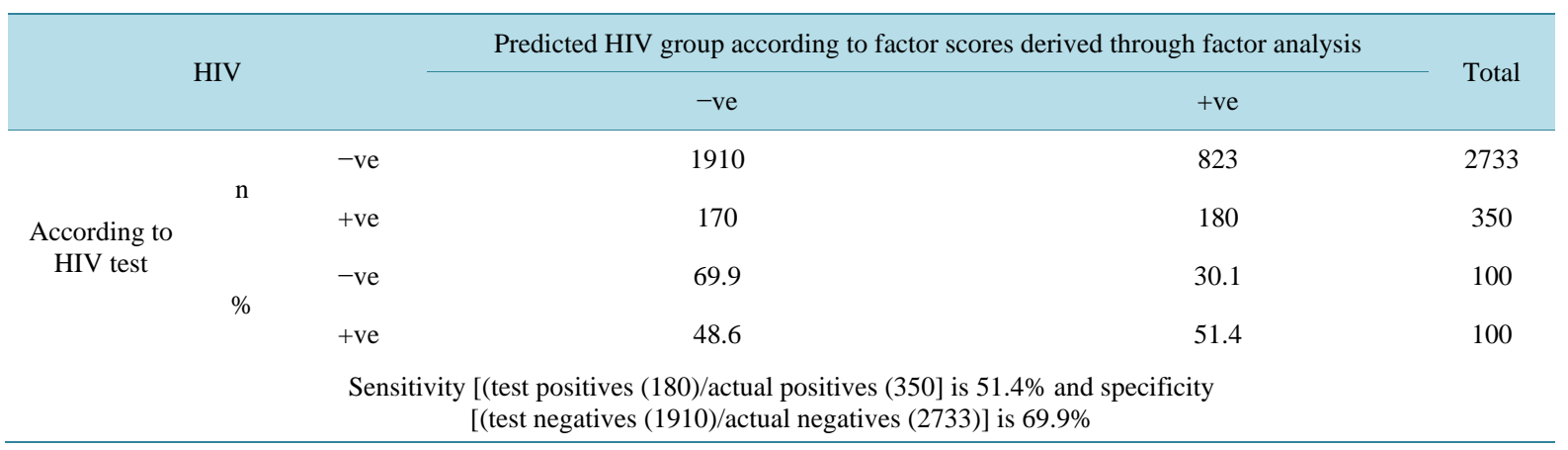

\section{Discussion}

In India, Andhra Pradesh is one of the high prevalence States with an estimated 16 per cent prevalence among commercial FSWs, ranging from 8 - 41 per cent. The present study reported $11.4 \%$ prevalence of HIV varying from district to district. Half of the FSWs in the present study had their first sexual debut at a very young age $(<25$ years). Similar observations were made in a survey carried out in 2006 [11].

The extent of condom use at with occasional clients reported in the present study was similar to an earlier report [11]. With HIV prevalence being high among FSWs, it is important to promote condom use in all types of sexual relationships, including regular, occasional partners and non commercial partners, since even non commercial partners might have risky sexual behaviours such as unprotected sex with multiple partners.

Analysis of behavioral patterns may offer benefit of summarizing behavior using a small number of factors. Yet, these may be potentially of greater relevance to educate the high risk population on more healthy behavior. Both PCA and cluster analysis are useful approaches for the assessment of behavioral patterns. A commonly-cited criticism of the two techniques is that these involve several subjective-but important—decisions, such as grouping of demographic and behaviors and possible transformations of variables. PCA involves decisions about the number of components to be retained and their subsequent labeling. Similarly, cluster analysis requires choices about the method of clustering and labeling of clusters. Another disadvantage of these techniques is that they generate patterns based on variation in the variables, with no guarantee that these patterns will be predictive of a particular health outcome. However, the techniques have the advantage that they are empirically derived and are, therefore, not limited by mere a priori knowledge.

In developing countries, studies on identifying behavioral patterns and their relationship with HIV status are scarce. Nine patterns among the variables considered explaining $62 \%$ of the total variability.

The first factor, labeled as having unpaid regular partners who accounted for $10.9 \%$ of the total variance followed by exposure to intervention $9.2 \%$, age \& duration of sex work 7.8\%, knowledge of HIV/AIDS and getting HIV testing done voluntarily $6.0 \%$ and about $5.0 \%$ each by the other factors.

The Socio demographic, behavioral and the biological parameters are highly correlated, and the classification methods based on univariate analysis may lead to flawed estimates [12]. Therefore, multivariate methods, such as factor analysis, represent an alternative approach to the evaluation of individual characteristics for the identification of patterns allows us to examine the effect of behavioral factors as whole and helps describe the association with HIV status. Moreover, it should be kept in minds that individuals behave significantly different, which are influenced by many factors, such as, socioeconomic, demographic and behavioral characteristics. Describing socio demographic, behavioral and biological patterns may be useful in developing community programmes for these high risk populations. Rather than changing the behavior, such programmes can be aimed at changing the behavior that is readily recognized by the target group. It is possible to find a smaller number of measures using factor analysis that would permit the identification of risk behaviors. The present data showed a considerable relationship between the socio demographic, behavioral and biological patterns and the HIV status. About $68 \%$ of FSWs were correctly classified as they were positive or negative for HIV test with sensitivity of 51.4\% and specificity of $70 \%$. The behavioral patterns that are extracted from the data obtained in the study may not be applicable to other populations with altogether different behavioral characteristics. 


\section{Conclusion}

The data allowed identification of patterns defined by factor analysis based on the data collected from FSWs. There exists a strong relationship between specific behavior patterns and HIV status among the high risk populations. These results will be useful for identifying people in the community with risk behaviors, related to HIV/AIDS, which will help the planners to develop suitable interventions for control and prevention of HIV/AIDS.

\section{Acknowledgements}

The authors thank the Director, National Institute of Nutrition for his support throughout the study and acknowledge the hard work and commitment of the IBBA Team at, Field Division and Microbiology Division. Financial support was provided by the Bill and Melinda Gates Foundation through Avahan, its India AIDS initiative.

\section{Conflict of Interest}

The authors have no conflict of interests.

\section{References}

[1] NACP (2012) Phase III State Fact Sheets. www.naco.gov.in

[2] ANC (2011) HIV Sentinel Surveillance 2010 Bulletin. Strategic Information Management Unit, APSACS, Andhra Pradesh.

[3] Chandrasekaran, P., Dallabetta, G., Loo, V., Mills, S., Saidel, T., Adhikary, R., Alary, M., Lowndes, C.M., Boily, M.C. and Moore, J., Avahan Evaluation Partners (2008) Evaluation Design for Large-Scale HIV Prevention Programmes: The Case of Avahan, the India AIDS Initiative. AIDS, 22, S1-S15. http://dx.doi.org/10.1097/01.aids.0000343760.70078.89

[4] (2009-10) National Summary Report, Round-2. Integrated Behavioral and Biological Assessment.

[5] Saidel, T., Adhikary, R., Mainkar, M., Dale, M., Loo, V., Rahman, M., et al. (2008) Baseline Integrated Behavioral and Biological Assessment among Most At-Risk Populations in Six High-Prevalent States of India: Design and Implementation Challenges. AIDS, 22, S17-S34. http://dx.doi.org/10.1097/01.aids.0000343761.77702.04

[6] (2011) Guidelines for Surveys of Populations at Risk of HIV Infections. Integrated Behavioral and Biological Assessment. Define the Study Population. 9.

[7] Mardia, K.V., Kent, J.M. and Bibby, J.M. (1980) Multivariate Analysis. Academic Press, London, 521 p.

[8] Kleinbaum, D.G., Kupper, L.L. and Muller, K.E. (1988) Variable Reduction and Factor Analysis. Applied Regression Analysis and Other Multivariable Methods. PWS Kent Publishing Co., Boston, 595-640.

[9] Aldenderfer, N.S. and Blashfield, R.K. (1984) Cluster Analysis. Sage Publication, Beverly Hills, 7-17.

[10] SPSS Version 19.0, IBM, Armonk.

[11] (2005-7) National Summary Report, Round-1. Integrated Behavioral and Biological Assessment.

[12] Hemalatha, R., Hari Kumar, R., Venkaiah, K., Srinivasan, K. and Brahmam, G.N.V. (2011) Prevalence of \& Knowledge, Attitude \& Practices towards HIV \& Sexually Transmitted Infections (STIs) among Female Sex Workers (FSWs) in Andhra Pradesh. Indian Journal of Medical Research, 134, 49-54. 